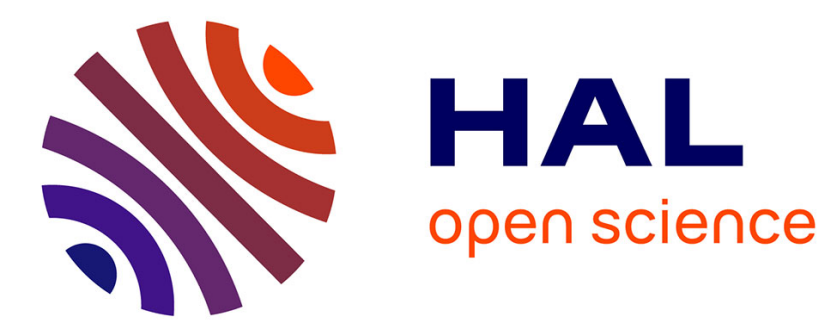

\title{
Combining high-flow nasal cannula oxygen and non-invasive ventilation for pre-oxygenation in the critically ill: is a double-pronged approach warranted?
}

Kris Bauchmuller, Alastair Glossop, Audrey de Jong, Samir Jaber

\section{- To cite this version:}

Kris Bauchmuller, Alastair Glossop, Audrey de Jong, Samir Jaber. Combining high-flow nasal cannula oxygen and non-invasive ventilation for pre-oxygenation in the critically ill: is a double-pronged approach warranted?. Intensive Care Medicine, 2017, 43 (2), pp.288 - 290. 10.1007/s00134-016-4635-6 . hal-01797617

\section{HAL Id: hal-01797617 \\ https://hal.science/hal-01797617}

Submitted on 11 Dec 2019

HAL is a multi-disciplinary open access archive for the deposit and dissemination of scientific research documents, whether they are published or not. The documents may come from teaching and research institutions in France or abroad, or from public or private research centers.
L'archive ouverte pluridisciplinaire HAL, est destinée au dépôt et à la diffusion de documents scientifiques de niveau recherche, publiés ou non, émanant des établissements d'enseignement et de recherche français ou étrangers, des laboratoires publics ou privés. 


\title{
Combining high-flow nasal cannula
} oxygen and non-invasive ventilation for pre-oxygenation in the critically ill:
is a double-pronged approach warranted?

\author{
Discussion on article "Apnoeic oxygenation via high-flow nasal cannula oxygen
} combined with non-invasive ventilation preoxygenation for intubation in hypoxaemic patients in the intensive care unit: the single-centre, blinded, randomised controlled OPTINIV trial"

Kris B. Bauchmuller ${ }^{1 *}$, Alastair J. Glossop ${ }^{1}$, Audrey De Jong ${ }^{2,3}$ and Samir Jaber ${ }^{2,3}$

\section{Initial correspondence from Drs. Bauchmuller and Glossop}

We read with great interest the article by Jaber et al. [1] and commend the authors on their work. Intubation in the intensive care unit (ICU) remains a high-risk intervention, despite recent efforts to protocolise care and refine the individual steps of the induction process. Consequently, this study, which combines non-invasive ventilation (NIV) and high-flow nasal cannula (HFNC) oxygen administration, is welcome, more so in that it examines an intriguing concept and also raises several important questions.

The authors define severe hypoxaemia at inclusion as an arterial partial pressure of oxygen/fraction of inspired oxygen ratio (PFR) of $<300 \mathrm{mmHg}$, which conflicts with the acute respiratory distress syndrome (ARDS) Berlin definition of moderate and severe hypoxaemia as a PFR of $<200$ and $<100 \mathrm{mmHg}$, respectively. Whilst we acknowledge that the median baseline PFR ratio in their study was $122 \mathrm{mmHg}$, the inclusion of a more severely hypoxaemic group, representing greater physiological limitation, might have delivered greater discrimination between the different treatment modalities.
The authors acknowledge the uncertainty about the clinical relevance of a modest difference in the minimum peripheral oxygen saturations $\left(\mathrm{SpO}_{2}\right)$ during intubation between groups (median $\mathrm{SpO}_{2} 96$ vs. 100\%). However, a higher rate of significant hypoxaemia $(<80 \%)$ was noted in the sham HFNC group. Whilst the study did not record any episodes of $\mathrm{SpO}_{2}$ of $<92 \%$ at $5 \mathrm{~min}$ after intubation, it would be interesting to assess the absolute duration of severe hypoxaemia after induction and whether this time correlates with relevant clinical outcomes. Conversely, there is increasing evidence of detrimental effects of supra-normal arterial oxygenation for prolonged periods [2]. Median $\mathrm{SpO}_{2}$ at 30 min after intubation was $99 \%$ in both groups with interquartile ranges of $97-100 \%$ and $98-100 \%$, respectively. This may warrant further consideration in the design of a larger, outcome-centred trial of the proposed intervention.

Data were collected in a single centre with extensive experience in the delivery of NIV for pre-oxygenation and a protocol for induction of anaesthesia. Whilst we commend the authors for the meticulous attention to detail in delivering this intervention, the wider application of their findings may be limited. Their study addresses preoxygenation during rapid sequence induction (RSI) with a prolonged apnoea after administration of muscle relaxants. However, there is emerging evidence that gentle bag-mask ventilation during the apnoeic phase is safe and

\footnotetext{
*Correspondence: kris.bauchmuller@sth.nhs.uk ${ }^{1}$ Department of Critical Care, Sheffield Teaching Hospitals NHS Foundation Trust, Herries Road, Sheffield S5 7AU, UK Full author information is available at the end of the article
} 
not associated with increased pulmonary aspiration. In addition, a recent case series described successful combination of ventilator-delivered NIV pressure support and maintenance of apnoeic oxygenation with nasal cannulae during the period of laryngoscopy and intubation [3]. Whilst the evidence base for this is modest, we feel that the practice of gentle ventilation post-induction warrants future comparison against the classical RSI technique described in Jaber et al.s study.

We feel that this is an important study which contributes significantly to the current evidence pool. We hope that future studies will focus on severe hypoxaemia prior to enrolment, be adequately powered to assess important patient-centred outcomes, such as severe complication rates and mortality, and provide the opportunity to assess the effects on long-term cognitive dysfunction secondary to hypoxaemia in a vulnerable ICU population.

\section{Reply from Drs. Jaber and De Jong}

We wish to thank Drs. Glossop and Bauchmuller for their comments in response to our recently published article in Intensive Care Medicine [1]. As underlined by them, we included patients with severe hypoxemic acute respiratory failure in the OPTINIV trial. The criteria of acute hypoxemic respiratory failure used in the OPTINIV trial were those generally applied in published studies in the field of preoxygenation [3-5]. We did not include exclusively patients with ARDS, but rather patients with hypoxemic acute respiratory failure (hypoxemia and tachypnea; see criteria in the article [1]) who required invasive mechanical ventilation whatever the etiology (see Table 1 of Jaber et al. [1]). However, all included patients presented tachypnea with a PFR of $<100$ and $<200 \mathrm{mmHg}$ in 50 and $28 \%$ of cases, respectively.

We agree with the accurate observation of Drs. Glossop and Bauchmuller that it would be interesting to assess the absolute duration of severe hypoxemia after induction and whether this time correlates with relevant clinical outcomes. The absolute duration of severe hypoxemia for the six patients in our study who experienced oxygen saturation of $<80 \%$ was $340,390,30,60,70$ and $260 \mathrm{~s}$, respectively. However, this number of patients was too low to draw any conclusion on clinical outcomes according to the duration of severe hypoxemia. We agree that supra-normal arterial oxygenation for prolonged periods could have potential detrimental effects. However, the length of preoxygenation and apneic oxygenation did not last for $>15$ min overall when the intubation was difficult. We believe that the benefits of these interventions in terms of decreasing severe hypoxemia events which can lead to cardiac arrest, cerebral anoxia and death outweigh the potential risks of administering high doses of oxygen during a short period of time.
As accurately stated by Drs. Glossop and Bauchmuller, as well as in our discussion of the limitations of our study [1], the OPTINIV trial was a single-centre proofof-concept study, which could limit the generalization of the results. However, the OPTINIV method is a safe and simple procedure that can be easily applied. Drs. Glossop and Bauchmuller suggest that the practice of gentle ventilation post-induction during apnea could become the standard of care of a modified rapid sequence induction, but further evidence is still needed. Even in the case of gentle bag mask or NIV pressure support ventilation during apnoea following rapid sequence induction, this apnoeic ventilation has to be removed during the laryngoscopy. However, the laryngoscopy can last several minutes in the case of difficult intubation [5]. During the apnoeic laryngoscopic period, continuous administration of oxygenation via high-flow nasal oxygen is maintained after removal the facial mask (connected to the bag mask or NIV).

Finally, Drs. Glossop and Bauchmuller concluded that there is a need for future studies which will be adequately powered to assess important patient-centred outcomes. We do realize that, as underlined in the OPTINIV paper, our study is a proof-of-concept study with the aim to evaluate if apneic oxygenation combined with NIV preoxygenation would allow a decrease in oxygen desaturation during intubation in the ICU. Further large studies with long-term complications outcomes are needed.

\section{Author details}

${ }^{1}$ Department of Critical Care, Sheffield Teaching Hospitals NHS Foundation Trust, Herries Road, Sheffield S5 7AU, UK. ${ }^{2}$ Department of Anesthesiology and Critical Care Medicine B (DAR B), Saint-Eloi Hospital, University Teaching Hospital of Montpellier, 80 avenue Augustin Fliche, 34295 Montpellier, France. 3 INSERM U1046, CNRS UMR, 9214 Montpellier, France.

\section{Compliance with ethical standards}

\section{Conflicts of interest}

AJG has received honoraria and speaking fees from Armstrong Medical UK between 2014 and 2016. SJ reports receiving consulting fees from Drager, Hamilton, Maquet, and Fisher \& Paykel.

\section{References}

1. Jaber S, Monnin M, Girard M, Conseil M, Cisse M, Carr J, Mahul M, Delay JM, Belafia F, Chanques G, Molinari N, De Jong A (2016) Apnoeic oxygenation via high-flow nasal cannula oxygen combined with non-invasive ventilation preoxygenation for intubation in hypoxaemic patients in the intensive care unit: the single-centre, blinded, randomised controlled OPTINIV trial. Intensive Care Med 42:1877-1887. doi:10.1007/ s00134-016-4588-9

2. Girardis M, Busani S, Damiani E, Donati A, Rinaldi L, Marudi A, Morelli A, Antonelli M, Singer M (2016) Effect of conservative vs conventional 
oxygen therapy on mortality among patients in an intensive care unit: the oxygen-ICU randomized clinical trial. JAMA 316:1583-1589

3. Grant S, Khan F, Keijzers G, Shirran M, Marneros L (2016) Ventilator-assisted preoxygenation: protocol for combining non-invasive ventilation and apnoeic oxygenation using a portable ventilator. Emerg Med Australas $28: 67-72$
4. Vourc'h M, Asfar P, Volteau C (2015) High-flow nasal cannula oxygen during endotracheal intubation in hypoxemic patients: a randomized controlled clinical trial. Intensive Care Med 41:1538-1548

5. De Jong A, Molinari N, Conseil M (2014) Video laryngoscopy versus direct laryngoscopy for orotracheal intubation in the intensive care unit: a systematic review and meta-analysis. Intensive Care Med 40:629-639 